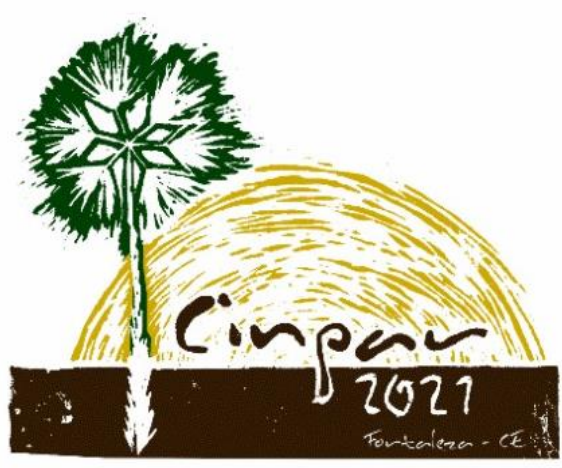

XVII Congresso Internacional sobre Patologia e Reabilitação das Construções

XVII Congreso Internacional sobre Patología y Rehabilitación de las Construcciones

XVII International Conference on Pathology and Constructions Rehabilitation

FORTALEZA (Brasil), 3 a 5 de junho de 2021

https://doi.org/10.4322/CINPAR.2021.110

\title{
Redistribuição de esforços em um reservatório de água devido à recalques nas fundações
}

\section{Forces transfer in a water storage tank due to foundations settlements}

\author{
Vinicius Tibes Caetano de SOUZA ${ }^{1}$, Gustavo SAVARIS ${ }^{2}$, Flavia de Fatima Emi MURAKAMI ${ }^{3}$, Carlos Eduardo \\ Tino BALESTRA ${ }^{4}$, Sérgio Antônio BRUM JUNIOR ${ }^{5}$, Fernando Nunes CAVALHEIRO ${ }^{6}$ \\ ${ }^{1}$ Universidade Tecnológica Federal do Paraná, Campus Toledo, Brasil, tibes_13@hotmail.com \\ 2 Universidade Tecnológica Federal do Paraná, Campus Toledo, Brasil, gsavaris@utfpr.edu.br \\ ${ }^{3}$ Universidade Tecnológica Federal do Paraná, Campus Curitiba, Brasil, flaviaemi21@yahoo.com.br \\ ${ }^{4}$ Universidade Tecnológica Federal do Paraná, Campus Toledo, Brasil, carlosbalestra@utfpr.edu.br \\ ${ }^{5}$ Universidade Tecnológica Federal do Paraná, Campus Toledo, Brasil, sergiojunior@utfpr.edu.br \\ ${ }^{6}$ Universidade Estadual do Oeste do Paraná, Campus Toledo, Brasil, fernandonunescavalheiro@hotmail.com
}

\begin{abstract}
Resumo: Os projetos estruturais geralmente são dimensionados considerando que as fundações sejam indeslocáveis. Contudo, as deformações do solo e consequente o deslocamento dos apoios ocasionam a redistribuição de cargas na superestrutura. Assim, o presente trabalho abordou o estudo da redistribuição dos esforços em um reservatório de água elevado submetido a recalques diferenciais. Um reservatório foi modelado em um programa computacional de análise estrutural, sendo utilizadas molas com diferentes coeficientes de rigidez sob os apoios, comparando as reações nos apoios da estrutura obtidas com um modelo de referência com apoios indeslocáveis. A análise da estrutura em apoios elásticos revelou uma maior redistribuição das cargas, indicando uma tendência de transferência de carga para os pilares mais próximos ao recalque. Aconselha-se que a verificação aqui realizada seja incorporada as rotinas usuais de projeto, pois essa consideração pode prever casos onde a superestrutura deverá ser repensada em razão de deslocamentos excessivos.
\end{abstract}

Palavras-chave: Interação solo-estrutura. Redistribuição de esforços. Reservatório. Recalques diferenciais

\begin{abstract}
Structural design practice generally considers that the foundations are restrained. However, soil strains and consequent foundations settlements provides a redistribution of loads on the superstructure. Thus, this work presents the forces redistribution in an elevated water storage tank submitted to differential settlements. A storage tank was modeled using structural analysis program, considering springs under the supports with different stiffness coefficients, comparing the forces obtained with a reference model, with restrained supports. The analysis of the structure on elastic supports revealed a greater redistribution of loads, indicating a tendency of load transfer to the columns closest to the settlement. It is advisable that the verification performed here be incorporated into the usual design routines, as this consideration may provide for cases where the superstructure should be redesigned due to excessive displacements.
\end{abstract}

Keywords: Soil structure interaction. Forces redistribution. Water storage tank. Differential settlements. 


\section{Introdução}

Nos projetos estruturais de edificações as cargas atuantes na fundação e o dimensionamento dos elementos estruturais de edifícios são geralmente realizados considerando a hipótese de que as fundações são indeslocáveiejam indeslocáveis. Da mesma forma os projetos de fundações são elaborados considerando apenas as propriedades geotécnicas do terreno e as cargas obtidas na análise estrutural, desprezando-se o efeito da rigidez da estrutura e considerando deslocamentos admissíveis de maneira que sejam compatíveis com a estrutura, para que não ocasionem danos que comprometam a estabilidade, a utilização ou a aparência estética.

Os recalques admissíveis constituem parte importante nas análises e projetos de fundações, definindo um limite a partir do qual se considera problemática a segurança ou o desempenho da estrutura (MILITITSKY, et al. 2006). Segundo Gusmão (2006), a ocorrência de recalques diferenciais causa o aparecimento de esforços secundários nos elementos estruturais, tais como tensões cisalhantes nas faces das paredes, surgimento de momentos negativos e positivos nos apoios periféricos e centrais da viga, respectivamente, e a redistribuição das cargas dos pilares.

O termo interação solo-estrutura (ISE) envolve uma área de estudo abrangente e pode incluir vários tipos de estruturas (prédios, pontes, túneis, silos, muros de arrimos, entre outras) e o solo sobre o qual são assentadas, sendo definida como um mecanismo de influência recíproca entre o sistema de fundação e a superestrutura, iniciando já na fase de construção e contínua até que a estrutura e o maciço de solos alcancem um estado de equilíbrio de tensões e deformações (COLARES, 2006).

Diversas pesquisas têm demonstrado que a adoção de apoios elásticos por meio da ISE conduz a esforços totalmente diferentes daqueles calculados considerando a fundação assentada sobre apoios fixos. Esses aspectos foram estudados por vários autores, citam-se aqueles publicados por Gusmão (1990; 1994; 2006), Goshy (1978), Meyerhof (1953), Fonte et al. (1994). Esta redistribuição depende, entre outras coisas, da rigidez relativa estrutura-solo e da deformada de recalques da edificação.

A compatibilidade das deformações na ISE tem como resultado uma tendência para uniformização dos recalques, a depender da rigidez do conjunto solo-estrutura. Este efeito, ao diminuir a curvatura da deformada dos recalques, reduz as distorções angulares da edificação e pode sim evitar o aparecimento de danos por fissuramento (GUSMÃO, 1990).

Gusmão (1990) e Savaris et al. (2011) ressaltam a relevância de se considerar em projetos de edificações o efeito da redistribuição de cargas nos elementos estruturais porque a mesma, quando ocorrer de forma significativa, pode provocar danos na superestrutura, tais como fissuras em vigas, lajes e esmagamento de pilares.

Com o aumento da velocidade de processamento dos computadores e o desenvolvimento de métodos numéricos refinados, a consideração da interação solo-estrutura em projetos vem sendo viabilizada, permitindo uma análise mais real do comportamento mecânico da estrutura e considerando a deformabilidade do solo, permitindo que patologias decorrentes dos recalques diferenciais sejam reduzidas ou evitadas.

Com o intuito de analisar o efeito da interação solo-estrutura em reservatório de água elevado, este trabalho apresenta uma análise numérica desta estrutura, toda em concreto armado, avaliando os esforços nos elementos estruturais em duas situações: considerando apoios indeslocáveis, usualmente realizada em projetos, e após a ocorrência de recalques nas bases dos pilares.

\section{Metodologia}

\subsection{Modelagem da estrutura}

A estrutura em concreto armado foi dimensionada por Freitas (2007) e consiste em um reservatório de água elevado de $125 \mathrm{~m}^{3}$ (Figura 1), com 4 metros de altura e 6,30 m de diâmetro, constituído de laje de fundo e paredes com 12 e $18 \mathrm{~cm}$ de espessura respectivamente, e uma tampa com espessura de $6 \mathrm{~cm}$, suportado por 6 pilares, com $12 \mathrm{~m}$ de altura e seção transversal quadrada de $30 \mathrm{~cm}$ de lado. Na altura média dos pilares foi 
dimensionada uma viga de travamento, para redução do comprimento de flambagem dos elementos, com seção transversal de $20 \mathrm{~cm} \times 40 \mathrm{~cm}$.

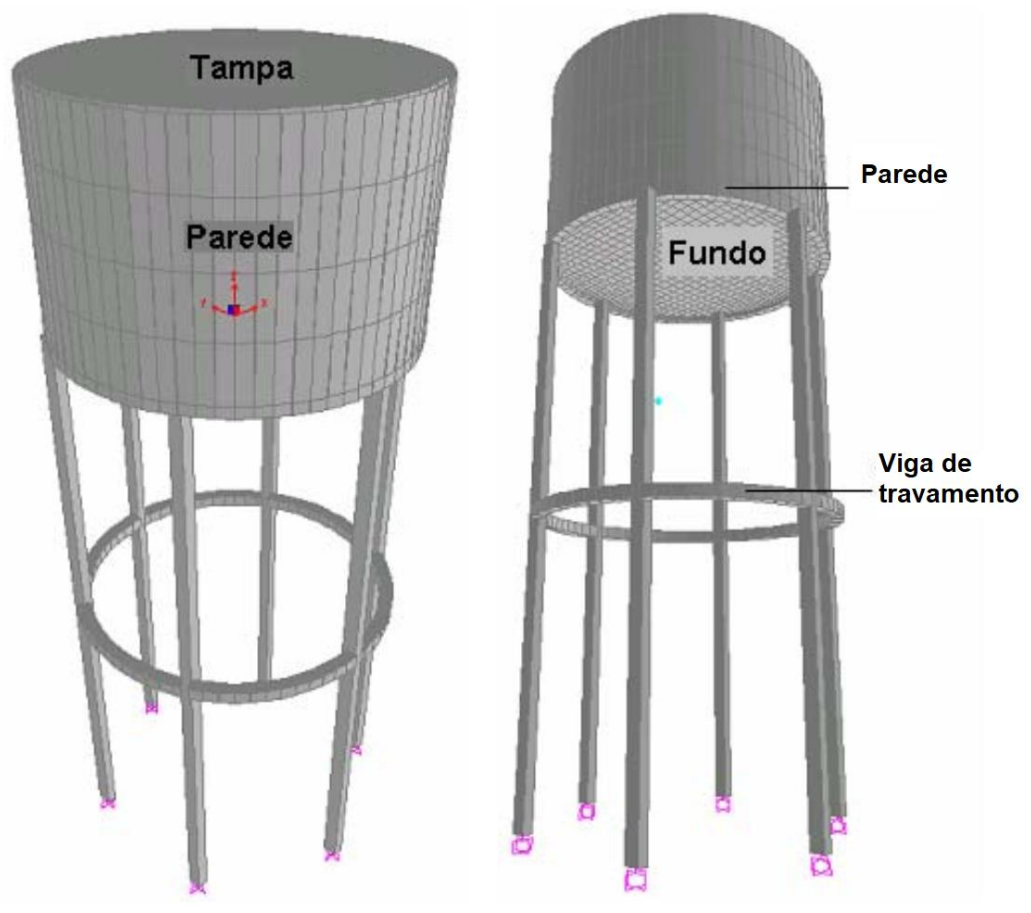

Figura 1 - Modelo computacional do reservatório (Freitas, 2007)

O reservatório tem como carregamentos solicitantes o peso próprio dos elementos de concreto armado (tampa, parede, fundo, pilares e tirantes de contraventamentos), considerado igual a $25 \mathrm{kN} / \mathrm{m}^{3}$, o peso da água (considerando a situação do reservatório totalmente cheio) e o empuxo gerado nas paredes.

Considerando que o objetivo do trabalho foi de avaliar os efeitos dos recalques em situação estática foram desconsideradas as cargas acidentais de manutenção e de vento.

Para implementação no progama de análise estrutural, o carregamento de água foi considerado como uniforme na face superior da laje de fundo, igual a $40 \mathrm{kN} / \mathrm{m}^{2}$, e triangular na face interna das paredes, iniciando em zero no topo da parede e $40 \mathrm{kN} / \mathrm{m}^{2}$ em sua base.

O modelo tridimensional foi composto por elementos de barra para discretizar o perfil das vigas e das colunas, com seis graus de liberdade por nó, sendo três translações e três rotações nas direções $x, y$ e $z$, e para discretizar as lajes e a parede de concreto foram adotados elementos de casca plana, definido por quatro nós, espessura e considera efeitos de membrana e/ou flexão, com seis graus de liberdade por nó, sendo três translações e três rotações nas direções $x, y$ e $z$.

Para o material concreto foram adotados para resistência à compressão, módulo de elasticidade e coeficiente de Poisson os valores de $30 \mathrm{MPa}, 30,7 \mathrm{GPa}$ e 0,2 , respectivamente.

\subsection{Análise da estrutura}

Análise estática linear da estrutura foi realizda considerando duas situações condições de apoios distintas:

$\mathrm{Na}$ primeira análise as reações nos apoios e os esforços nos elementos estruturais foram obtidos considerando os apoios dos pilares engastados no solo, impedindo os deslocamentos e as rotações nas direções $x, y$ e $z$.

Para a segunda análise o apoio indeslocável sob o pilar P1 foi substituído por uma mola com comportamento elástico linear, enquanto os demais pilares continuaram indeslocáveis, na Figura 2 é apresentada a disposição dos pilares e das vigas de travamento em planta. 


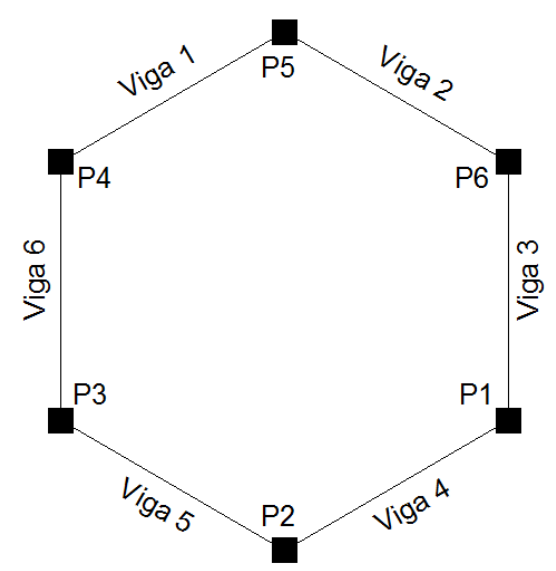

Figura 2 - Disposição dos pilares e vigas de travamento

De acordo com Barros (2005) distorções angulares da ordem de 1/1000 são responsáveis por pequenas fissuras em painéis externos e 1/500 é o limite de segurança para estruturas em que não são admissíveis fissuras. Assim, os valores dos coeficientes de rigidez de mola para este estudo foram determinados considerando a distorção angular gerada pelo deslocamento vertical do pilar P1 em relação aos pilares P2 e P6, que distam 3 metros. Para ocorrência de distorções angulares de 1/1000 e 1/500 são necessários recalques no apoio do pilar $\mathrm{P} 1 \mathrm{da}$ ordem de 3 e $6 \mathrm{~mm}$, respectivamente.

A partir das reações verticais obtidas nos apoios para a condição de apoios indeslocáveis, o coeficiente de rigidez linear da mola $(K)$ foi calculado pela relação entre a reação no apoio e o recalque, resultando em $K 1$ igual a $52,5 \mathrm{MN} / \mathrm{m}$ e $K 2$ igual a $26,25 \mathrm{MN} / \mathrm{m}$, sendo adotado o valor de $K 3$ igual a zero representando o colapso do solo sob o elemento de fundação.

Para cada condição de apoio foi realizada a análise da estrutura e comparadas as magnitudes das reações nos apoios, sendo avaliada a redistribuição de cargas dos pilares utilizando o coeficiente de redistribuição $(F R)$, definido como:

$$
\left.F R=\frac{R_{c r}-R_{i}}{R_{i}}\right) 100
$$

onde $R_{i}$ é a reação no apoio do pilar $i$ calculada considerando os apoios indeslocáveis e $R_{c r}$ é a reação no apoio do pilar $i$ calculada considerando o recalque do pilar P1. O coeficiente $F R$ representa em porcentagem o acréscimo ou alívio de carga no apoio devido aos recalques.

\section{Resultados e discussão}

\subsection{Efeito da ISE nas cargas dos pilares}

As reações nos apoios da estrutura, obtidas nos modelos considerando os apoios indeslocáveis e com a adoção de molas sob o pilar P1, são apresentadas na Tabela 1.

Tabela 1 - Reações nos apoios dos pilares (kN)

\begin{tabular}{|c|c|c|c|c|}
\hline Pilar & Indeslocável & K1 & K2 & K3 \\
\hline P1 & 321,86 & 154,49 & 96,56 & 0,00 \\
\hline P2 & 321,86 & 444,17 & 482,79 & 556,17 \\
\hline P3 & 321,86 & 289,67 & 281,95 & 258,78 \\
\hline P4 & 321,86 & 308,99 & 305,12 & 301,26 \\
\hline P5 & 321,86 & 289,67 & 281,95 & 258,78 \\
\hline P6 & 321,86 & 444,17 & 482,79 & 556,17 \\
\hline
\end{tabular}


As reações obtidas na análise considerando os apoios fixos apresentaram valores iguais para todos os apoios, resultado este esperado por se tratar de uma estrutura com simetria geométrica e de carregamentos. Verifica-se que conforme diminui a rigidez da mola sob o apoio sob o pilar P1 ocorre a redução da reação vertical neste pilar, podendo ser atribuída à rigidez da superestrutura, que restringe o deslocamento vertical do pilar, redistribuindo a carga aos demais.

Devido à simetria da estrutura, verifica-se que as reações nos apoios 2 e 6 são iguais, assim como nos apoios 3 e 5 . Os apoios 2 e 6 sofreram acréscimos de carga conforme ocorrem os recalques no apoio 1 , por estarem mais próximos, enquanto nos apoios 3 e 5 verificou-se a redução da carga, como consequencia de um efeito de alavanca. Para o apoio 4, se verifica uma pequena variação nas reações.

Os valores apresentados na Tabela 2 representam a porcentagem de redução de carga, para valores negativos, ou de acréscimo, para valores positivos, calculados considerando o coeficiente $F R$ (Equação 1).

Tabela 2 - Coeficiente de redistribuição de cargas (\%)

\begin{tabular}{|c|c|c|c|}
\hline Pilar & K1 & K2 & K3 \\
\hline P1 & $-52,0$ & $-70,0$ & $-100,0$ \\
\hline P2 & 38,0 & 50,0 & 72,8 \\
\hline P3 & $-10,0$ & $-12,4$ & $-19,6$ \\
\hline P4 & $-4,0$ & $-5,2$ & $-6,4$ \\
\hline P5 & $-10,0$ & $-12,4$ & $-19,6$ \\
\hline P6 & 38,0 & 50,0 & 72,8 \\
\hline
\end{tabular}

Para o coeficiente de mola $K 1$ verifica-se um alívio de $52 \%$ na carga axial do pilar P1, entretanto esse efeito não é tão relevante quanto o acréscimo de carga, o qual pode gerar danos estruturais. Verfica-se que nos pilares P2 e P6 ocorre um acréscimo de $38 \%$ em relação ao modelo usualmente adotado no dimensionamento, com apoios indeslocáveis. Apesar deste coeficiente de mola ser definido a partir de uma distorção angular pequena, dependendo do fator de segurança adotado pelo projetista podem ocorrer falhas estruturais com essa magnitude de acréscimo.

Quando os recalques ocorrem com maior magnitude, como nos casos de coeficientes de mola $K 2$ e $K 3$, verifica-se nos pilares P2 e P6 acréscimos de carga de $50 \%$ e 72,8\%, respectivamente. Considerando que os pilares são elementos solicitados à flexão composta, aumento de carga desta magnitude provavelmente acarretaria no colapso da estrutura.

\section{Conclusões}

Com a análise estrutura de um reservatório de concreto armado submetido à recalques em um dos apoios verifica-se a importância da consideração da interação solo-estrutura.

A análise da estrutura em apoios elásticos revelou a redistribuição das cargas, indicando significativa transferência de carga, sobrecarregando os pilares mais próximos e reduzindo a carga dos pilares mais afastados.

Recomenda-se a adoção da análise da interação solo-estrutura nas rotinas usuais de projeto, pois essa consideração pode reduzir patologias ocasionadas por recalques das fundações.

\section{Agradecimentos}

Os autores gostariam de agradecer à Universidade Tecnológica Federal do Paraná, campus Toledo, e ao Grupo de Pesquisa em Materiais e Estruturas (GPMAES), pelo suporte à pesquisa.

\section{Referências Bibliográficas}


BARROS, R.A. (2005) Avaliação do comportamento de fundações em estaca hélice através de medidas de recalques. Dissertação de Mestrado. Universidade Estadual do Norte Fluminense Darcy Ribeiro, Campos dos Goytacazes - RJ, Brasil.

COLARES, G.M. (2006) Programa para análise da interação solo-estrutura no projeto de edifícios. Dissertação de Mestrado. Escola de Engenharia de São Carlos, Universidade de São Paulo, Brasil.

FONTE, A.O.C., PONTES FILHO, I.D.S., JUCÁ, J.F.T. (1994) Interação solo-estrutura em edifícios altos. In. X COBRAMSEG. Anais..., Foz do Iguaçu, PR. v.1, p 237-46.

FREITAS, J.P. (2007) Dimensionamento estrutural de um reservatório elevado de água para um condomínio residencial. de Conclusão de Curso de Graduação. Universidade Estadual do Norte Fluminense Darcy Ribeiro, Campos dos Goytacazes - RJ, Brasil.

GOSHY, B. (1978) Soil Foundation Structure Interaction. Journal of the Structural Division, ASCE, 104 (ST5), 749-761.

GUSMÃO, A.D. (1990) Estudo da interação solo-estrutura e sua influência em recalques de edificações. Dissertação de Mestrado. COPPE/UFRJ, Rio de Janeiro, Brasil.

GUSMÃO, A.D. (2006) Desempenho de fundações de edifícios. In: Palestras XIII COBRAMSEG, pp 215-240, Curitiba.

MEYERHOF, G.G. (1953) Some recent foudation research and its application to design. The Structural Engineering. Londres.

MILITITSKY, J., CONSOLI, N.C., SCHNAID, F. (2006) Patologia das fundações. São Paulo: Oficina de Textos.

SAVARIS, G., HALLAK, P.H., MAIA, P.C.A. (2011) Understanding the Mechanism of Static Soil-Structure Interaction - A Case Study. Soils and Rocks, 34 (3), 195-206. 